\title{
Method for measurement of pulsating flow of liquids and gases
}

\author{
Zh.A. Dayev \\ LLP «Technopark «Zerek» Baishev University Aktobe \\ Research Laboratory "Information and measuring systems" \\ Aktobe, Kazakhstan \\ Tel. / Fax: +7 (7132) 57-89-75 \\ e-mail: zhand@yandex.ru
}

The objective of the present article is to find a relationship for a pulsating flow of an ideal liquid. Such a relationship must serve as a basic model for the solution of the problem of measuring the velocity of flows of actual liquids and gases. A method of variable pressure drop based on constrictions will be considered in the article, as it is one of the simplest and most commonly employed methods used for the measurement of flows and quantities of liquids and gases.

By $[1,2,3,4]$, as the frequency of pulsations increases the local component of the acceleration of the liquid introduces the basic contribution to the measurement error. Let us model a process involving the flow of a liquid, taking into account this component. The movement of an ideal liquid is described by Euler's equation [5]:

$$
\frac{\partial V}{\partial t}+V \cdot \nabla V=-\frac{1}{\rho} \nabla p,
$$

where $V, \rho$ and $p$ are the velocity, density, and pressure of the liquid, respectively; $t$, time; and $\nabla$ is the Hamiltonian operator.

In solving different types of problems, Eq. (1) is complemented with an equation of continuity, represented by the law of conservation of matter:

$$
\frac{\partial \rho}{\partial t}+\nabla(\rho V)=0
$$

It is known that $j=\rho V$ is the density of the flow, from which the quantity of liquid traveling in unit time through a unit of area situated perpendicular to the velocity is determined [5]. Due to difficulties involved in integration of Eqs. (1) and (2), when solving different types of problems it is assumed that the liquid is incompressible and that its motion is steady-state, hence the local components of the derivatives of the velocity and density are set equal to zero, after which the equation is easily integrated. Following integration, Eqs. (1) and (2) become standard equations that serve as the basis of many methods used in the measurement of flow rates and the quantity of liquids and gases, for example, Bernoulli methods and the method of continuity of a flow.

We will find a solution of Eq. (1) for a one-dimensional flow of a pulsating ideal liquid and assume that within the scope of the problem its density is constant. For this purpose, we first replace the partial derivatives by infinitely small differences and rewrite (1) in the following form:

$$
\frac{d V}{d t}+V \frac{d V}{d x}=-\frac{1}{\rho} \frac{d p}{d x}
$$

We next divide Eq. (3) by the convective component of the derivative of the velocity. Reducing similar terms, we obtain

$$
\frac{d x}{V d t}+1=-\frac{1}{\rho} \frac{d p}{V d V} .
$$

In (4), we replace the increment $d x$ by a finite characteristic length $L$ of the channel and the time increment by a finite interval of time, which we denote $1 / f$, and then rewrite (4) as

$$
\left(\frac{L f}{V}+1\right) V d V=-\frac{d p}{\rho} .
$$

where $f$ is the frequency of the pulsations of the flow.

We next remove the parentheses and integrate (5), after which we deduce the expression

$$
\frac{V^{2}}{2}\left(2 \frac{L f}{V}+1\right)+\frac{p}{\rho}=\text { const . }
$$


where $S h=L f / V$ is the Strouhal number, which is a measure of the nonsteadiness of a pulsating flow of an ideal liquid.

We rewrite (6) in the form of a solution of Eq. (1) for a pulsating flow in the following form:

$$
\frac{V^{2}}{2}(2 S h+1)+\frac{p}{\rho}=\text { const } .
$$

Thus, (7) is the final equation of motion of a pulsating flow of an ideal liquid. Carrying out analogous arguments, we find a relation for the equation of continuity of a flow for the case of a pulsating flow of liquid:

$$
j(S h+1)=\text { const } .
$$

For $S h \gg>1$, the flow is assumed to be unsteady and the local component of the acceleration begins to dominate its convective component. The latter circumstance is taken into account in (7) and (8) by a nonzero Strouhal number. For $S h<<1$ or $S h \rightarrow 0$, the two expressions (7) and (8) become Bernoulli equations and the equation of continuity for a steady flow.

A graph that expresses the dependence of the density of the flow on the velocity of the flow is presented in Fig. 1. From the graph, it follows that ignoring the frequency of the pulsations may lead to additional errors in measurements of the flow, while the density of the velocity of the flow grows significantly with a growth in frequency. We may derive relationships that implement a technique for measuring a pulsating flow of a liquid on the basis of Eqs. (7) and (8).

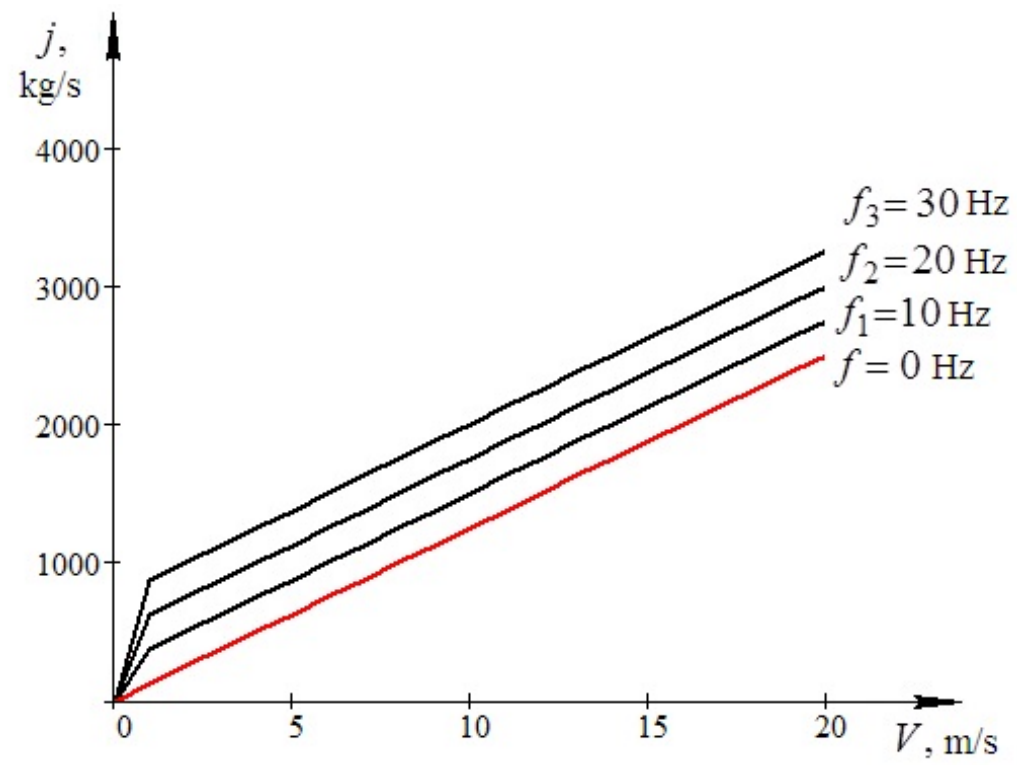

Fig. 1. Comparative dependence of density of flow on velocity for different values of the frequency of the pulsations: 1) $f=0 \mathrm{~Hz}$; 2) $f 1=10 \mathrm{~Hz}$; 3) $f 2=20 \mathrm{~Hz}$; 4) $f 3=30 \mathrm{~Hz}$

The process by which a pulsating flow travels is depicted in Fig. 2. To compile the motion equations, we will consider two sections, using the methods of hydrodynamics and geometric similitude. From (7), we find, using Fig. 2, the following equation:

$$
V_{2}=\sqrt{\frac{\psi}{(1+\xi)\left(2 S h_{2}+1\right)-\mu^{2} \beta^{4}\left(\frac{S h_{2}+1}{S h_{1}+1}\right)^{2}}} \sqrt{\frac{2 \Delta p}{\rho}}=\alpha \sqrt{\frac{2 \Delta p}{\rho}} .
$$

where $V_{1}$ and $V_{2}$ are the flow rates in the first and second sections, respectively; $S h_{1}$ and $S h_{2}$, Strouhal numbers in the corresponding sections; $p_{1}$ and $p_{2}$, pressure of the fluid before and after the constriction; and $\xi$, coefficient of hydraulic resistance.

The flow rate in the first section may be expressed from (8) as follows:

$$
V_{1}=\mu \beta^{2} \frac{S h_{2}+1}{S h_{1}+1} V_{2},
$$

where $\mu$ is a coefficient that takes into account the constriction of the current, and $\beta$ is the ratio of the diameter of the constriction $d$ to the diameter of the pipeline $D$. 
We introduce into (9) and (10) the pressure tap coefficient $\psi=\Delta p^{*} / \Delta p$, where $\Delta p^{*}=p_{1}-p_{2}$ is the pressure drop in the constriction and $\Delta p$ the pressure drop fed to the input of the measurement transducer. We write an expression for the velocity of the liquid in the second section as

$$
V_{2}=\sqrt{\frac{\psi}{(1+\xi)\left(2 S h_{2}+1\right)-\mu^{2} \beta^{4}\left(\frac{S h_{2}+1}{S h_{1}+1}\right)^{2}}} \sqrt{\frac{2 \Delta p}{\rho}}=\alpha \sqrt{\frac{2 \Delta p}{\rho}},
$$

where $\alpha$ is the flow coefficient; the Strouhal number, which is related to the pulsating nature of the movement, occurs in the flow coefficient.

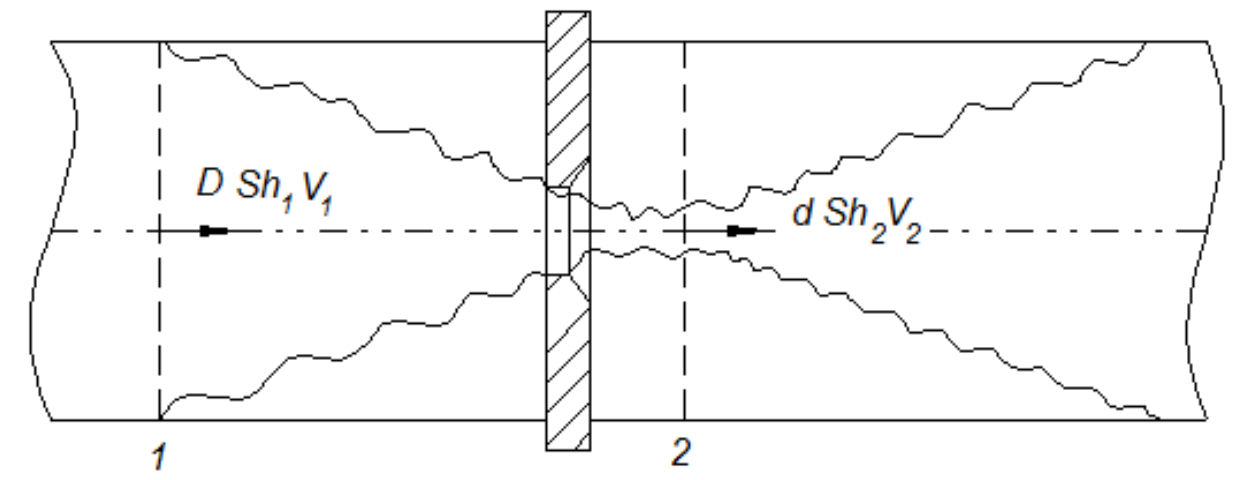

Fig. 2. Movement of the pulsating fluid flow through the constriction: 1, 2) first and second sections, respectively

Let us now derive an equation for the flow coefficient of the inlet $E$, which may be written in terms of the ratio of the kinetic energy of the flow to the potential energy, expressed in the form of the pressure drop in the constriction. We determine this coefficient by jointly solving Eqs. (7) and (8):

$$
E=\frac{1}{\sqrt{1-\beta^{4}\left(\frac{2 S h_{1}+1}{2 S h_{2}+1}\right)\left(\frac{S h_{2}+1}{S h_{1}+1}\right)^{2}}} .
$$

Dividing the flow coefficient in Eq. (11) by Eq. (12), we obtain the efflux coefficient of the constriction for a pulsating flow:

$$
C=\frac{\alpha}{E}=\sqrt{\frac{\psi\left(1-\beta^{4}\right) k_{o} k_{S h}^{2}}{(1+\xi)\left(2 S h_{2}+1\right)-\mu^{2} \beta^{4} k_{S h}^{2}}},
$$

where $k_{O}=\frac{2 S h_{1}+1}{2 S h_{2}+1}$ and $k_{S h}=\frac{S h_{2}+1}{S h_{1}+1}$.

Thus, in this case the efflux coefficient $C$ is also a function of the Strouhal number.

It is easily noted that if the Strouhal number vanishes, the quantities determined from formulas (11)-(13) assume the traditional values for steady-state motion of a liquid. Consequently, for pulsating flows the traditional Reader-Harris or Stolz equations used to find the efflux coefficient require some modification through the introduction of the Strouhal number.

We may now write an expression for use in determining the flow rate of a liquid:

$$
Q=C E \frac{\pi d^{2}}{4} \sqrt{\frac{2 \Delta p}{\rho}} .
$$

The distinctive feature of the proposed method consists in the presence of the Strouhal number, which occurs in the efflux coefficient and takes into account the nonsteady nature of the flow. Consequently, for nonsteady flows of liquid it is necessary to have corresponding expressions for the efflux coefficients and the rate of entry. In the case of measurements of the velocity of a nonsteady flow of gas, a corresponding coefficient of expansion must also 
be found. The basic assumptions of the proposed method must be verified by simultaneously measuring the frequency of the pulsations of the flow through a particular section of the pipeline and the velocity of the flow, i.e., by establishing a dependence of the efflux coefficient of the constriction on the Strouhal number. As the frequency of the pulsations increases for one and the same flow rate, the recorded flow rate of the substance will increase, acquiring an additional acceleration (cf. Fig. 1). After experimental data have been obtained, the method may be extended to actual liquids and gases.

Main results of this paper were published in the papers $[6,7]$.

\section{REFERENCES}

1. Kremlevsky P.P. Flow meters and meters the quantity of substances. Directory. L.: Engineering, 1989. $701 \mathrm{p}$.

2. Tweten D., Nored M., Brun K. The Physics of Pulsations // Gas Machinery Conference. Albuquerque, 2008. pp. 1 - 16.

3. Shemer L., Wygnanski I., Kit E. Pulsating flow in a pipe // J. Fluid. Mech. 1985. vol. 153. pp. 313 - 337.

4. Gajan P., Mottram R.C., Hebrard P., Andriamihafy H., Platet * B. The influence of pulsating flows on orifice plate flowmeters // Flow measurement and Instrumentation. 1992. vol. 3. pp. 118 - 129.

5. LD Landau, EM Lifshitz, Theoretical physics. T. 6. Hydrodynamics. - M.: Nauka, 1986. 736 p.

6. Daev Zh.A. A method for the measurement of a pulsating flow of liquid // Measurement Techniques. 2016. vol. 59. pp. 243 - 246.

7. Daev Zh.A. A Model of combination of automated measuring system for pulsating flow and the amount of substance // Instruments. 2016. №2. pp 21 - 23. 